\title{
Characterizations of the Exponentiated Marshall-Olkin Discrete Uniform Distribution
}

\author{
Zadan P. H. Mason ${ }^{1}$, Mahrokh Najaf ${ }^{2}$ \\ ${ }^{1}$ University School of Milwaukee, River Hills, WI 53217, USA \\ ${ }^{2}$ Department of Mathematical and Statistical Sciences, Marquette University, Milwaukee, WI 53201-1881, USA \\ Correspondence: Mahrokh Najaf, Department of Mathematical and Statistical Sciences, Marquette University, Milwau- \\ kee, WI 53201-1881, USA
}

Received: August 11, 2021 Accepted: September 6, 2021 Online Published: September 25, 2021

doi:10.5539/ijsp.v10n6p1 URL: https://doi.org/10.5539/ijsp.v10n6p1

\section{Abstract}

In this short paper, we consider certain characterizations of the Exponentiated Marshall-Olkin Discrete Uniform (EMODU) distribution introduced by Gharib et al. (2017) to complete in some manner, the authors' work.

Keywords: discrete distribution, characterizations, conditional expectation, hazard function, reverse hazard function

\section{Introduction}

The cumulative distribution function (cdf), $F(x)$, probability mass function (pmf), $f(x)$, hazard function, $h_{F}(x)$, and reverse hazard rate function, $r_{F}(x)$, are give by

$$
\begin{aligned}
F(x ; \alpha, \theta, \gamma) & =\left(\frac{x+1}{\alpha \theta+(1-\theta)(x+1)}\right)^{\gamma}, & x=0,1, \ldots \alpha, \\
f(x ; \alpha, \theta, \gamma) & =\left(\frac{x+1}{\alpha \theta+(1-\theta)(x+1)}\right)^{\gamma}-\left(\frac{x}{\alpha \theta+(1-\theta) x}\right)^{\gamma}, & x=0,1, \ldots, \alpha, \\
h_{F}(x) & =\frac{1-\left(\frac{x}{\alpha \theta+(1-\theta) x}\right)^{\gamma}}{1-\left(\frac{x+1}{\alpha \theta+(1-\theta)(x+1)}\right)^{\gamma}}-1, & x=0,1, \ldots, \alpha, \\
r_{F}(x) & =1-\left(\frac{x(\alpha \theta+(1-\theta)(x+1))}{(x+1)(\alpha \theta+(1-\theta) x)}\right)^{\gamma}, & x=0,1, \ldots, \alpha,
\end{aligned}
$$

Respectively, where $\alpha$, a positive integer and $\beta>0, \gamma>0$ are parameters.

\subsection{Remark}

In defining the cdf of EMODU, Gharib et al. did not make the distinction we have made here for $x=0$.

\section{Characterization Results}

We present our characterizations via three subsections $2.1,2.2$ and 2.3. In the following subsections, we let $I=\{0,1, \ldots, \alpha\}$. 2.1 Characterizations of EMODU in Terms of the Conditional Expectation of Certain Function of the Random Variable

\subsubsection{Proposition}

Let $X: \Omega \rightarrow I$ be a random variable. The pmf of $X$ is (2) if and only if 


$$
\begin{aligned}
& E\left\{\left[\left(\frac{X+1}{\alpha \theta+(1-\theta)(X+1)}\right)^{\gamma}+\left(\frac{X}{\alpha \theta+(1-\theta) X}\right)^{\gamma}\right] \mid X \leq k\right\} \\
= & \left(\frac{k+1}{\alpha \theta+(1-\theta)(k+1)}\right)^{\gamma}, \quad k \in I .
\end{aligned}
$$

Proof: If $X$ has pmf (2), then the left-hand side of (5), using the telescoping series property, will be

$$
\begin{aligned}
& (F(k))^{-1} \sum_{x=0}^{k}\left\{\left[\left(\frac{x+1}{\alpha \theta+(1-\theta)(x+1)}\right)^{2 \gamma}-\left(\frac{x}{\alpha \theta+(1-\theta) x}\right)^{2 \gamma}\right]\right\} \\
= & \left(\frac{k+1}{\alpha \theta+(1-\theta)(k+1)}\right)^{-\gamma}\left\{\left(\frac{k+1}{\alpha \theta+(1-\theta)(k+1)}\right)^{2 \gamma}\right\} \\
= & \left(\frac{k+1}{\alpha \theta+(1-\theta)(k+1)}\right)^{\gamma} .
\end{aligned}
$$

Conversely, if (5) holds, then

$$
\begin{aligned}
& \sum_{x=0}^{k}\left\{\left[\left(\frac{x+1}{\alpha \theta+(1-\theta)(x+1)}\right)^{\gamma}+\left(\frac{x}{\alpha \theta+(1-\theta) x}\right)^{\gamma}\right] f(x)\right\} \\
= & F(k)\left(\frac{k+1}{\alpha \theta+(1-\theta)(k+1)}\right)^{\gamma} \\
= & {[F(k+1)-f(k+1)]\left(\frac{k+1}{\alpha \theta+(1-\theta)(k+1)}\right)^{\gamma}, }
\end{aligned}
$$

where we have used $F(k)=F(k+1)-f(k+1)$.

From (5), we also have

$$
\begin{aligned}
& \sum_{x=0}^{k+1}\left\{\left[\left(\frac{x+1}{\alpha \theta+(1-\theta)(x+1)}\right)^{\gamma}+\left(\frac{x}{\alpha \theta+(1-\theta) x}\right)^{\gamma}\right] f(x)\right\} \\
= & F(k+1)\left(\frac{k+2}{\alpha \theta+(1-\theta)(k+2)}\right)^{\gamma} .
\end{aligned}
$$

Subtracting (6) from (7), we come up with

$$
\begin{aligned}
& F(k+1)\left[\left(\frac{k+2}{\alpha \theta+(1-\theta)(k+2)}\right)^{\gamma}-\left(\frac{k+1}{\alpha \theta+(1-\theta)(k+1)}\right)^{\gamma}\right] \\
= & {\left[\left(\frac{k+2}{\alpha \theta+(1-\theta)(k+2)}\right)^{\gamma}\right] f(k+1), }
\end{aligned}
$$

and hence

$$
r_{F}(k+1)=\frac{f(k+1)}{F(k+1)}=1-\left(\frac{(k+1)(\alpha \theta+(1-\theta)(k+2))}{(k+2)(\alpha \theta+(1-\theta)(k+1))}\right)^{\gamma}
$$

which, considering (4), suggests that $X$ has pmf (2). 


\subsection{Characterization of EMODU Based on Hazard Function}

\subsubsection{Proposition}

If $X: \Omega \rightarrow I$ is a random variable, then the pmf of $X$ is (2) if and only if its hazard rate function satisfies the difference equation

$$
h_{F}(k+1)-h_{F}(k)=\frac{1-\left(\frac{k+1}{\alpha \theta+(1-\theta)(k+1)}\right)^{\gamma}}{1-\left(\frac{k+2}{\alpha \theta+(1-\theta)(k+2)}\right)^{\gamma}}-\frac{1-\left(\frac{k}{\alpha \theta+(1-\theta) k}\right)^{\gamma}}{1-\left(\frac{k+1}{\alpha \theta+(1-\theta)(k+1)}\right)^{\gamma}},
$$

$k \in I$, with the boundary condition $h_{F}(0)=\frac{(\alpha \theta+1-\theta)^{\gamma}}{(\alpha \theta+1-\theta)^{\gamma}-1}-1$.

Proof: If $X$ has pmf (2), then obviously (8) holds. Now, if (8) holds, then for every $x \in I$, we have

$$
\begin{aligned}
& \sum_{k=0}^{x-1}\left\{h_{F}(k+1)-h_{F}(k)\right\} \\
= & \sum_{k=0}^{x-1}\left\{\frac{1-\left(\frac{k+1}{\alpha \theta+(1-\theta)(k+1)}\right)^{\gamma}}{1-\left(\frac{k+2}{\alpha \theta+(1-\theta)(k+2)}\right)^{\gamma}}-\frac{1-\left(\frac{k}{\alpha \theta+(1-\theta) k}\right)^{\gamma}}{1-\left(\frac{k+1}{\alpha \theta+(1-\theta)(k+1)}\right)^{\gamma}}\right\},
\end{aligned}
$$

or

$$
h_{F}(x)-h_{F}(0)=\frac{1-\left(\frac{x}{\alpha \theta+(1-\theta) x}\right)^{\gamma}}{1-\left(\frac{x+1}{\alpha \theta+(1-\theta)(x+1)}\right)^{\gamma}}-\frac{(\alpha \theta+1-\theta)^{\gamma}}{(\alpha \theta+1-\theta)^{\gamma}-1} .
$$

Considering the fact that $h_{F}(0)=\frac{(\alpha \theta+1-\theta)^{\gamma}}{(\alpha \theta+1-\theta)^{\gamma}-1}-1$, from the last equation we have

$$
h_{F}(x)=\frac{1-\left(\frac{x}{\alpha \theta+(1-\theta) x}\right)^{\gamma}}{1-\left(\frac{x+1}{\alpha \theta+(1-\theta)(x+1)}\right)^{\gamma}}-1,
$$

which, considering (3), suggests that $X$ has pmf (2).

\subsection{Characterization of EMODU Based on Reverse Hazard Function}

\subsubsection{Proposition}

If $X: \Omega \rightarrow I$ is a random variable, then the pmf of $X$ is (2) if and only if its reverse hazard rate function satisfies the difference equation

$$
\begin{aligned}
& r_{F}(k+1)-r_{F}(k)=\left(\frac{k(\alpha \theta+(1-\theta)(k+1))}{(k+1)(\alpha \theta+(1-\theta) k)}\right)^{\gamma} \\
& -\left(\frac{(k+1)(\alpha \theta+(1-\theta)(k+2))}{(k+2)(\alpha \theta+(1-\theta)(k+1))}\right)^{\gamma},
\end{aligned}
$$

$k \in I$, with the boundary condition $r_{F}(0)=1$.

Proof: If $X$ has pmf (2), then obviously (9) holds. Now, if (9) holds, then for every $x \in I$, we have

$$
\begin{aligned}
& \sum_{k=0}^{x-1}\left\{r_{F}(k+1)-r_{F}(k)\right\} \\
= & \sum_{k=0}^{x-1}\left\{\left(\frac{k(\alpha \theta+(1-\theta)(k+1))}{(k+1)(\alpha \theta+(1-\theta) k)}\right)^{\gamma}-\left(\frac{(k+1)(\alpha \theta+(1-\theta)(k+2))}{(k+2)(\alpha \theta+(1-\theta)(k+1))}\right)\right\},
\end{aligned}
$$


or

$$
r_{F}(x)-r_{F}(0)=-\left(\frac{x(\alpha \theta+(1-\theta)(x+1))}{(x+1)(\alpha \theta+(1-\theta) x)}\right)^{\gamma} .
$$

In view of the condition $r_{F}(0)=1$, from the above equation we have

$$
r_{F}(x)=1-\left(\frac{x(\alpha \theta+(1-\theta)(x+1))}{(x+1)(\alpha \theta+(1-\theta) x)}\right)^{\gamma},
$$

which, in view of (4), implies that $X$ has pmf (2).

\subsection{Remark}

Sandhya and Prasanth (2014) considered the following discrete distribution, called Marshall-Olkin Discrete Uniform (MODU), with pmf given by

$$
f(x ; \alpha, \theta)=\frac{\alpha \theta}{(\alpha \theta+(1-\theta) x)(\alpha \theta+(1-\theta)(x+1))}, \quad x=0.1, \ldots, \alpha .
$$

The EMODU is an extension of MODU for $\gamma=1$.

\section{References}

Gharib, M., Mohammed, B. I., \& Aghel, W. E. R. (2017). The exponentiated Marshall-Olkin discrete uniform distribution with application in survival analysis. International Journal of Modern Engineering Research, 7(8), 34-48.

Sandhya, E., \& Prasanth, C. B. (2014). Marshall-Olkin discrete uniform distribution. Journal of Probability Article ID 979312, 10 pages. https://doi.org/10.1155/2014/979312

\section{Copyrights}

Copyright for this article is retained by the author(s), with first publication rights granted to the journal.

This is an open-access article distributed under the terms and conditions of the Creative Commons Attribution license (http://creativecommons.org/licenses/by/4.0/). 